\title{
Índices morfofisiológicos de alface produzidas em diferentes substratos sob ambiente protegido
}

A alface é considerada a hortaliça folhosa mais importante no Brasil. A produção em casa de vegetação ou ambiente protegido, utilizando substrato, chamado também de cultivo sem solo, vem ganhando destaque, no cenário mundial, devido a necessidade de conservação do solo. Desta forma, a busca por materiais alternativos para compor substratos é necessária e relevante, devendo levar em conta a aptidão agroindustrial de cada região com a finalidade de permitir o aproveitamento de resíduos que estão disponíveis abundantemente e a redução dos custos de produção. $\mathrm{O}$ objetivo de avaliar resíduos industriais utilizados como substratos, e seus efeitos no desenvolvimento e produção da alface cultivada em ambiente protegido. Esse experimento foi desenvolvido na Universidade Federal Rural da Amazônia, campus de Paragominas, na área experimental de Horticultura. Utilizou-se o delineamento experimental inteiramente casualisados com quatro tratamentos (palha de arroz, resíduo de soja, mistura= palha de arroz $(35 \%)+$ resíduo de soja $(35 \%)+$ areia preta (30\%) e areia preta como testemunha). Foram avaliadas as seguintes características: número de folhas, Peso $(\mathrm{kg})$, Peso raiz $(\mathrm{g})$, ciclo, comprimento do caule ( $\mathrm{cm}$ ), produtividade econômica $(\mathrm{kg})$, área foliar (AR) (cm2), índice de área foliar (IAF), Razão de Área Foliar (RAF) (cm2 g-1), área foliar específica (AFE) (cm2 g-1), razão de peso das folhas (RPF), quantidade de água na parte aérea (QAPA), peso específico foliar (PEF) $(\mathrm{g} \mathrm{cm} 2)$. Todos os dados obtidos foram analisados estatisticamente através da análise de variância, com teste F. Realizou-se o teste de Tukey (5\%) para a comparação de médias. O melhor substrato para produção de alface, sem solo, em casa de vegetação, é o resíduo de soja, pois nesse substrato a cultivar veneranda apresentou a maior produtividade e os melhores índices morfofisiológicos, demostrando a possibilidade da utilização desse resíduo industrial, como substrato alternativo.

\section{Morpho-physiological indexes of lettuce produced on different substrates under protected environment}

\begin{abstract}
Lettuce is considered the most important leafy vegetable in Brazil. The production in a greenhouse or protected environment, using substrate, also called cultivation without soil, has been gaining prominence in the world scenario, due to the need for soil conservation. Thus, the search for alternative materials to compose substrates is necessary and relevant, taking into account the agro-industrial aptitude of each region in order to allow the use of waste that is abundantly available and the reduction of production costs. The objective of evaluating industrial residues used as substrates, and their effects on the development and production of lettuce grown in a protected environment. This experiment was developed at the Federal Rural University of the Amazon, campus of Paragominas, in the experimental area of Horticulture. A completely randomized design was used with four treatments (rice straw, soy residue, mixture $=$ rice straw $(35 \%)+$ soy residue $(35 \%)+$ black sand (30\%) and black sand as a control). The following characteristics were evaluated: number of leaves, Weight (kg), Root weight (g), cycle, stem length $(\mathrm{cm})$, economic productivity $(\mathrm{kg})$, leaf area (RA) $(\mathrm{cm} 2)$, leaf area index (IAF), Leaf Area Ratio (RAF) (cm2 g-1), specific leaf area (AFE) (cm2 g-1), leaf weight ratio (RPF), amount of water in the aerial part (QAPA), weight leaf specific (PEF) $(\mathrm{g} \mathrm{cm} 2)$. All data obtained were analyzed statistically through analysis of variance, with the $\mathrm{F}$ test. The Tukey test (5\%) was used to compare means. The best substrate for lettuce production, without soil, in a greenhouse, is the soy residue, because in this substrate the cultivar veneranda showed the highest productivity and the best morphophysiological indexes, demonstrating the possibility of using this industrial residue as an alternative substrate.
\end{abstract}

Keywords: Cultivation without soil; Vegetables; Lactuca sativa L.; Rice straw.

Topic: Desenvolvimento, Sustentabilidade e Meio Ambiente

Reviewed anonymously in the process of blind peer.
Received: 10/08/2020

Approved: 21/09/2020
Michelane Silva Santos Lima (D)

Universidade Estadual Paulista Júlio Mesquita Filho, Brasil http://lattes.cnpq.br/6818368804286499 http://orcid.org/0000-0002-5844-5833 michelanesilva12@gmail.com

\section{Luciana da Silva Borges (iD)}

Universidade Federal Rural da Amazônia, Brasil http://lattes.cnpq.br/4533722536181534 http://orcid.org/0000-0002-1194-6411 luciana.borges@ufra.edu.br

Luana Keslley Nascimento Casais (iD Universidade Federal Rural de Pernambuco, Brasil http://lattes.cnpq.br/8075409193627849 http://orcid.org/0000-0001-7197-5524 luana.casais@gmail.com

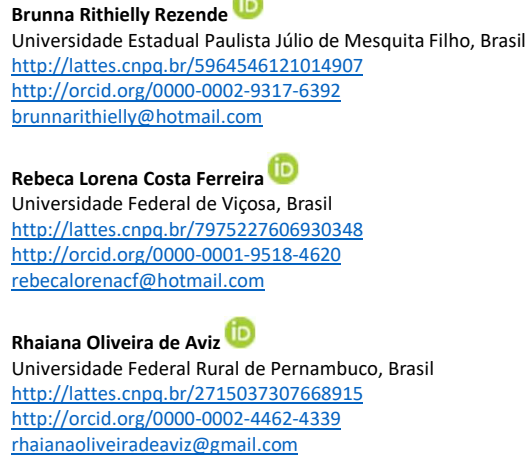

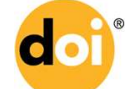

DOI: 10.6008/CBPC2179-6858.2020.005.0048
Referencing this:

LIMA, M. S. S.; BORGES, L. S.; CASAIS, L. K. N.; REZENDE, B. R.; FERREIRA, R. L. C.; AVIZ, R. O.; PONCE, F. S.; FREITAS, L. S.. Índices morfofisiológicos de alface produzidas em diferentes substratos sob ambiente protegido. Revista Ibero Americana de Ciências Ambientais, v.11, n.5, p.531-538, 2020. DOl:

http://doi.org/10.6008/CBPC2179-6858.2020.005.0048 


\section{INTRODUÇÃO}

A alface é considerada a hortaliça folhosa mais importante no Brasil, com grande importância econômica e social na agricultura. Seu cultivo é feito de maneira intensiva e geralmente praticado pela agricultura familiar, responsável pela geração de cinco empregos diretos por hectare (ALENCAR et al., 2012; FREITAS et al., 2013).

A hortaliça é produzida durante o ano todo e em todas as regiões do país. A região Amazônica possui uma precipitação média de aproximadamente $2300 \mathrm{~mm}$ (FISCH et al., 1996), com isso, durante a estação chuvosa o grande volume de água pode ser prejudicial ao cultivo da folhosa, visto que, ocasiona o encharcamento dos solos e consequentemente a perda de folhas, prejudicando assim a produtividade, deste modo, o cultivo em ambiente protegido se mostra uma alternativa viável para solucionar o problema, pois, permite a obtenção do produto final com boa qualidade mesmo sob condições ambientais desfavoráveis (MAGGI et al., 2006; PURQUERIO et al., 2007).

Contudo, a produção em ambiente protegido pode ocasionar prejuízos aos solos, principalmente, em função do seu uso intensivo com a realização de cultivos sucessivos, que podem levar ao aumento na incidência de patógenos na área de produção, além disso, a aplicação de altas doses de fertilizantes aliado a falta de lixiviação podem ocasionar o acúmulo de sais na superfície que resultam em dados a produção de alface (BLANCO et al., 2008; AZEVEDO et al., 2018).

Desta forma, o cultivo em substrato, também chamado cultivo sem solo, existe como uma alternativa para substituir o uso do solo e vem ganhando destaque no cenário mundial, devido a necessidade de diminuir os impactos ambientais na produção. Nesse sistema, o substrato utilizado é fator primordial, pois ele propiciará o desenvolvimento das plantas (CABRAL et al., 2011). A eficiência de um substrato é determinada por suas propriedades físicas, químicas e biológicas (LODOLINE et al., 2017). Além disso, é essencial que o insumo tenha baixo custo e boa disponibilidade.

Nesse contexto, a busca por materiais alternativos para compor substratos é necessária e relevante, devendo levar em conta a aptidão agroindustrial de cada região com a finalidade de permitir o aproveitamento de resíduos que estão disponíveis abundantemente e a redução dos custos de produção. Desta forma, este estudo tem o objetivo de avaliar resíduos industriais utilizados como substratos, e seus efeitos no desenvolvimento e produção da alface cultivada em ambiente protegido.

\section{MATERIAIS E MÉTODOS}

O experimento foi conduzido na área experimental de Horticultura e no laboratório multifuncional pertencente a Universidade Federal Rural da Amazônia, campus de Paragominas. O município de

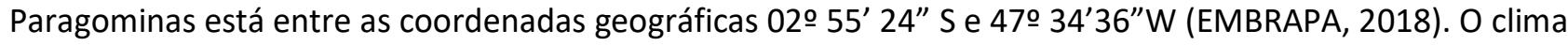
da região é do tipo Aw, segundo a classificação de Köppen, isto é, tropical chuvoso com estação seca bem definida, com temperatura média anual de 26,5 ㄷ. A umidade relativa do ar varia de $70 \%$ a 90\% (RODRIGUES et al., 2002). 
Para plantio utilizou-se sementes do cultivar de alface Veneranda, a semeadura foi realizada em bandejas de poliestireno expandido de 128 células, preenchidas com substrato comercial. As bandejas foram dispostas sobre bancadas de madeira coberto com sombrite, sendo transplantadas para local definitivo quando as mudas apresentaram quatro folhas definitivas, aos 32 dias após a semeadura

As mudas foram acondicionadas em vasos plásticos com dimensões de altura de $15 \mathrm{~cm}$ e diâmetro de $18 \mathrm{~cm}^{2}$, com capacidade de 1,5 L, sendo colocada 1 muda por vaso, que foram dispostas em bancadas em uma estrutura de ambiente protegido, coberto com filme plástico de polietileno. As médias das temperaturas mínima, máxima e média obtidas no interior da estrutura foram de 24; 33 e 28ํㅡ, respectivamente.

Os substratos utilizados nesse estudo foram palha de arroz, resíduo de soja, mistura composta por palha de arroz (35\%) + resíduo de soja (35\%) +areia preta (30\%) e testemunha constituída de areia preta, provenientes de industrias da região. Os substratos palha de arroz (Tabela 1), resíduo de soja (Tabela 2) e areia preta (Tabela 3) foram submetidos a análises químicas, conforme os resultados descritos a seguir, afim de verificar os nutrientes composto em cada substrato estudado.

Tabela 1: Resultados da análise química do substrato Palha de arroz.

\begin{tabular}{|c|c|c|c|c|c|c|c|c|c|}
\hline P Total & $\mathrm{F}$ & $\mathrm{Ca}$ & $\mathrm{Mg}$ & $\mathrm{Fe}$ & $\mathrm{Al}$ & $\mathrm{Cu}$ & $\mathrm{Zn}$ & $\mathrm{Mn}$ & $\mathrm{Na}$ \\
\hline & 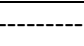 & ----'-- & $-\%$ & --. & -------- & \multicolumn{4}{|c|}{---------------------------- ppm ------------------------ } \\
\hline 0,4 & 0,08 & 0,04 & 0,05 & 2,67 & 5,22 & 0 & 58,06 & 99,38 & 273,81 \\
\hline B & M.O. & Umidade & $\mathrm{S}$ & N Total & Cinzas & $\mathrm{pH}(\mathrm{CaCl} 2)$ & Mo & Co & Densidade \\
\hline \multicolumn{7}{|c|}{ 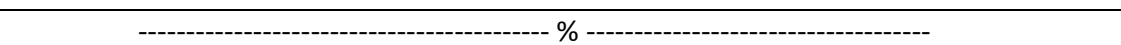 } & \multicolumn{2}{|c|}{----------- ppm --------- } & $\mathrm{g} / \mathrm{cm} 3$ \\
\hline 0,01 & 57,34 & 14,73 & 0 & 0,47 & 42,66 & 4,8 & 8,67 & 0 & 0,2 \\
\hline
\end{tabular}

Tabela 2: Resultados das análises químicas do substrato Resíduo de soja.

\begin{tabular}{|c|c|c|c|c|c|c|c|c|c|}
\hline P Total & $\mathrm{F}$ & $\mathrm{Ca}$ & $\mathrm{Mg}$ & $\mathrm{F}$ & $\mathrm{Al}$ & $\mathrm{Cu}$ & $\mathrm{Zn}$ & $\mathrm{Mg}$ & $\mathrm{Na}$ \\
\hline \multicolumn{6}{|c|}{ 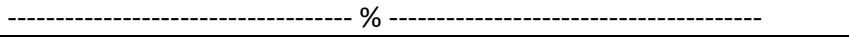 } & \multicolumn{4}{|c|}{ 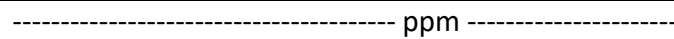 } \\
\hline 1,12 & 1,05 & 0,89 & 0,36 & 1,28 & 4,12 & 5,57 & 90,29 & 75,31 & 287,36 \\
\hline B & M.O. & Umidade & Enxofre & N Total & Cinzas & $\begin{array}{l}\mathrm{pH} \\
(\mathrm{CaCl} 2)\end{array}$ & Mo & Co & Densidade \\
\hline \multicolumn{7}{|c|}{ 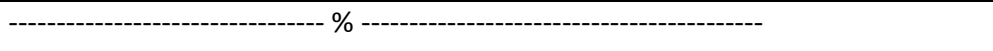 } & \multicolumn{2}{|c|}{------ ppm ----- } & $\mathrm{g} / \mathrm{cm} 3$ \\
\hline 0,01 & 63,65 & 10,33 & 0 & 2,16 & 36,35 & 7,7 & 9,75 & 2,22 & 0,41 \\
\hline
\end{tabular}

Tabela 3: Resultados das análises químicas do substrato Areia preta (Testemunha).

\begin{tabular}{|c|c|c|c|c|c|c|c|c|c|c|c|}
\hline$P$ & M.O. & COT & $\begin{array}{l}\mathrm{pH} \\
(\mathrm{CaCl} 2)\end{array}$ & $\begin{array}{l}\mathrm{pH} \\
\text { (SMP) }\end{array}$ & K & $\mathrm{Ca}$ & Mg & $\begin{array}{l}\mathrm{H}^{\circ}+\mathrm{Al} \\
3\end{array}$ & $\mathrm{Al}^{3}$ & $\mathrm{H}^{\circ}$ & C.T.C. \\
\hline $\mathrm{mg} / \mathrm{dm}^{3}$ & \multicolumn{4}{|c|}{$----\mathrm{g} / \mathrm{dm}^{3}$--- } & \multicolumn{7}{|c|}{ - } \\
\hline 22 & 20 & 12 & 4,7 & 5,9 & 1,6 & 15 & 4 & 47 & 6 & 41 & 67,7 \\
\hline \multirow[t]{2}{*}{ S.B. } & $\mathrm{S}$ & V\% & $\mathrm{K}$ na CTC & $\mathrm{Ca}$ & $\mathrm{Mg}$ na & Al na & na & $\mathrm{m} \%$ & $\mathrm{Ca} / \mathrm{K}$ & $\mathrm{Ca} / \mathrm{Mg}$ & $\mathrm{Mg} / \mathrm{K}$ \\
\hline & & & & CTC & CTC & CTC & CTC & & & & \\
\hline $\mathrm{mmolc} / \mathrm{dm}^{3}$ & \multicolumn{2}{|c|}{$--\mathrm{mg} / \mathrm{dm}^{3}--$} & --------- & --------- & ---------. & \% --- & ------- & ------ & & & \\
\hline 20,6 & 45 & 30 & 2,4 & 22,2 & 5,9 & 8,9 & 60,7 & 22,56 & 9,4 & 3,8 & 2,5 \\
\hline
\end{tabular}

Utilizou-se um delineamento experimental inteiramente casualisado com quatro tratamentos (palha, resíduo de soja, mistura composta palha de arroz + resíduo de soja + areia preta e testemunha constituído apenas por areia preta), e cinco repetições, contendo uma planta por vaso. O sistema de irrigação adotado foi o localizado por aspersão, utilizando-se mangueiras, e as irrigações foram realizadas diariamente em dois horários (manhã e tarde). O controle das plantas invasoras foi realizado manualmente a cada 7 dias. 
A adubação de plantio foi definida com base nas recomendações de Yuri et al. (2016), de acordo com as análises dos substratos, aplicou-se $3 \mathrm{~g} / \mathrm{m}^{-2}$ de ureia; $1,6 \mathrm{~g} / \mathrm{m}^{-2}$ de Cloreto de Potássio ( $56 \%$ de $\mathrm{K}_{2} \mathrm{O}$ ); $6 \mathrm{~g}$ / $\mathrm{m}^{-2}$ de superfosfato simples. Para adubação de cobertura utilizou-se adubação mineral foliar, utilizando-se 2 $\mathrm{ml}$ para $1 \mathrm{~L}$ de água, sendo aplicado com borrifador em cada vaso a cada 7 dias.

As plantas foram colhidas quando atingiram o máximo de desenvolvimento vegetativo, antes de iniciarem o processo de pendoamento, efetuada nas primeiras horas da manhã, sendo selecionadas e transportadas imediatamente ao laboratório, continuadamente, as plantas foram lavadas em água corrente para retirada do calor de campo e grandes impurezas, em seguida, foram colocadas sob papel absorvente para a retirada de excesso de água.

As características avaliadas foram: número de folhas, peso da planta (g), peso da raiz (g), comprimento do caule, ciclo e produtividade econômica. Foi realizado também a análise de crescimento, obtido a partir do índice de área foliar (IAF), razão de área foliar (RAF), área foliar específica (AFE), razão de peso das folhas (RPF), quantidade de água na parte aérea (QAPA), peso específico foliar (PEF) e área foliar (AF). Os dados foram analisados e interpretados estatisticamente a partir das análises de variância, com teste F. Quando houve significância para o fator foi aplicado teste de Tukey (5\%) para a comparação de médias. Todas as análises realizadas foram feitas com software SISVAR (FERREIRA, 2011).

\section{RESULTADOS E DISCUSSÃO}

De acordo com os resultados das análises estatísticas, observou-se que houve diferenças significativas do cultivar veneranda produzida em diferentes substratos, considerando as variáveis em estudo (Tabela 4). Para o parâmetro peso da planta, houve efeito significativo, onde o melhor resultado foi observado para o tratamento à base de resíduo de soja apresentando $67 \mathrm{~g}$, resultado superior aos demais substratos. Lima et al. (2018) avaliando a qualidade e produtividade de cultivares de alface conduzidas em campo no sudeste paraense, obteve peso $30 \mathrm{~g}$ para esta cultivar, resultado inferior aos obtidos nesse estudo para todos os substratos, indicando ser um tipo de cultivo promissor para produção de alface.

Tabela 4: Comparação de Peso da planta (PP), Peso da Raiz (PR), Número de folhas (NF), Comprimento do caule (CC) e Produtividade (PD) de alface produzidas em diferentes substratos sob ambiente protegido.

\begin{tabular}{llllll}
\hline SUBSTRATOS & PP $(\mathrm{g})$ & PR $(\mathrm{g})$ & NF & CC $(\mathrm{cm})$ & PD \\
\hline Mistura & $39 \mathrm{~b}$ & $7,89 \mathrm{~b}$ & $36,20 \mathrm{a}$ & $7,17 \mathrm{a}$ & $2.36 \mathrm{~b}$ \\
Testemunha & $36 \mathrm{~b}$ & $2,89 \mathrm{c}$ & $21,46 \mathrm{c}$ & $6,12 \mathrm{~b}$ & $2.15 \mathrm{~b}$ \\
Resíduo de soja & $67 \mathrm{a}$ & $17,19 \mathrm{a}$ & $33,60 \mathrm{a}$ & $3,77 \mathrm{c}$ & $4.00 \mathrm{a}$ \\
Palha & $39 \mathrm{~b}$ & $2,89 \mathrm{c}$ & $29,06 \mathrm{~b}$ & $5,93 \mathrm{~b}$ & $2.37 \mathrm{~b}$ \\
\hline CV & 5,21 & 13,30 & 5,42 & 7,77 & 5,28
\end{tabular}

Médias seguidas por letras iguais na coluna não diferem entre si pelo Tukey à $5 \%$ de probabilidade.

Para o peso da raiz os resultados diferiram estatisticamente, o tratamento resíduo de soja se sobressaiu dos demais apresentando 17,19 g. Segundo Bezerra (2013) a raiz é um órgão que possui as funções básicas de sustentação, reserva e absorção de água e nutrientes, desta forma torna-se importante ser estudada e relacionada com o desempenho da cultura, o que justifica o maior resultado de peso das plantas cultivadas nesse substrato. Casais et al. (2018), analisando o resíduo de soja, para produção de mudas de 
jambu, destacam o uso desse substrato alternativo, porém tem poucas informações. No entanto, esses autores ressaltam que é importante que aumentem as pesquisas com uso desse material, pois tem possibilidade de ser usado em alternância aos produtos comerciais, que aumentam significativamente os custos de produção.

Em relação a variável número de folhas, foi encontrado maior resultado para o para o substrato resíduo de soja. Lima et al. (2018), também avaliou o número de folhas e obteve resultado de 29,87 para a cultivar Veneranda, resultado inferior ao obtido neste estudo para o tratamento resíduo de soja que apresentou média de 36,20 . Os resultados das plantas cultivadas em substrato resíduo de soja se sobressaíram quando comparado ao tratamento testemunha, este desempenho pode estar relacionado com a composição nutricional destes substratos. Casais et al. (2018) avaliando diferentes substratos, observou que o resíduo de soja mostrou ser um substrato ideal a ser usado por proporcionar as plantas de pimentão um maior crescimento e maior número de frutos em relação aos demais tratamento utilizados (palha de arroz e substrato comercial).

Com relação ao parâmetro comprimento do caule o tratamento resíduo de soja apresentou menor média, e os tratamentos mistura, testemunha e palha de arroz obtiveram médias superiores, apresentando 7,$17 ; 6,1$ e 5,93 cm respectivamente indicando que as plantas produzidas nesses substratos apresentaram baixa resistência ao pendoamento precoce, que tem como característica o aumento da espessura do caule, antes de atingir o ciclo característico da cultivar, este distúrbio é uma característica indesejável e pode ter influência de temperaturas elevadas. Durante a condução desta pesquisa, as temperaturas obtidas foram mínimas, máxima e média de $24 ; 33$ e 28ํ․ A faixa ideal de temperaturas para o crescimento da alface é variável de acordo com a cultivar, no entanto, deve ser entre 15 a $28{ }^{\circ} \mathrm{C}$, Souza et al. (2013) dizem que temperaturas muito elevadas podem ocasionar vários distúrbios fisiológicos, dentre eles o florescimento precoce e alteração na taxa de crescimento.

Quanto à comparação entre os substratos para o parâmetro produtividade, verificou-se que a melhor média pertence ao tratamento resíduo de soja de $4,00 \mathrm{~kg}$, e as menores médias corresponderam aos tratamentos mistura, testemunha e palha de arroz embora não se diferenciaram estatisticamente. Lima et al. (2018) obteve produtividade de $1,81 \mathrm{~kg}$ para a cultivar Veneranda, resultado inferior a todos os tratamentos nesse estudo que variou de 2,15 a $4,00 \mathrm{~kg}$.

Na Tabela 5, observa-se diferenças significativas entre os substratos para os parâmetros índice morfofisiológicos de Área foliar (AF), Área foliar especifica (AFE), razão de peso foliar (RPF), índice área foliar $(\mathrm{IAF})$, peso específico da folha (PEF), razão de área foliar (RAF) e quantidade de água na parte aérea (QAPA).

Para área foliar constatou-se que o tratamento resíduo de soja e palha de arroz foi superior aos tratamentos mistura e testemunha apresentando $1110,13 \mathrm{~cm}^{2}$ e $1156,67 \mathrm{~cm}^{2}$ respectivamente. A palha de arroz tem diversos benefícios se tratando de substrato. Aviz et al. (2019) verificando a adaptação de cultivares de alface às condições climáticas do sudeste paraense, avaliaram a área foliar e obtiveram resultados que variaram de 87,03 a 123,70 $\mathrm{cm}^{2}$, assim como Pereira Junior et al. (2020), que avaliaram produtividade de cultivares de alface produzidas em campo em período seco de Paragominas, e obteve 
médias de área foliar entre $61,52 \mathrm{~cm}^{2}$ à $86,94 \mathrm{~cm}^{2}$, esses resultados são inferiores aos obtidos nesse trabalho. Segundo Ibarra et al. (2001), a área foliar é um fator importante da planta para ser avaliado, quando as pesquisas relacionam nutrição e crescimento vegetal, uma vez que, afeta o acúmulo de matéria seca, e também disponibiliza informações, no que tange o metabolismo vegetal, capacidade fotossintética, rendimento e particularidade da colheita.

Tabela 5: Índices morfofisiológicos de crescimento, Área foliar (AF), Área foliar especifica (AFE), Razão de área foliar (RAF), Razão de peso foliar (RPF), Quantidade de água na parte aérea (QAPA), Índice área foliar (IAF) e Peso específico da folha (PEF), produzidas em diferentes substratos sob ambiente protegido.

\begin{tabular}{llllllll}
\hline Tratamentos & $\mathrm{AF}\left(\mathrm{cm}^{2}\right)$ & ÍÁF & $\operatorname{RAF}\left(\mathrm{cm}^{2} \mathrm{~g}^{-1}\right)$ & $\mathrm{RPF}$ & $\mathrm{AFE}\left(\mathrm{cm}^{2} \mathrm{~g}^{-1}\right)$ & $\operatorname{PEF}\left(\mathrm{g} \mathrm{cm}^{2}\right)$ & QAPA \\
\hline Mistura & $577,85 \mathrm{~b}$ & $32,43 \mathrm{~b}$ & $74,51 \mathrm{c}$ & $90,23 \mathrm{c}$ & $74,51 \mathrm{c}$ & $0,014 \mathrm{~b}$ & $385,00 \mathrm{~b}$ \\
Testemunha & $583,82 \mathrm{~b}$ & $32,10 \mathrm{~b}$ & $189,52 \mathrm{a}$ & $231,66 \mathrm{a}$ & $190,32 \mathrm{a}$ & $0,005 \mathrm{c}$ & $356,03 \mathrm{~b}$ \\
Resíduo de Soja & $1110,13 \mathrm{a}$ & $61,67 \mathrm{a}$ & $55,90 \mathrm{~d}$ & $63,80 \mathrm{c}$ & $55,90 \mathrm{c}$ & $0,018 \mathrm{a}$ & $647,70 \mathrm{a}$ \\
Palha & $1156,67 \mathrm{a}$ & $64,09 \mathrm{a}$ & $143,37 \mathrm{~b}$ & $158,89 \mathrm{~b}$ & $143,37 \mathrm{~b}$ & $0,007 \mathrm{c}$ & $387,29 \mathrm{~b}$ \\
\hline $\mathrm{CV}$ & 5,45 & 5,46 & 7,31 & 15,20 & 17,84 & 14,46 & 5,33 \\
\hline
\end{tabular}

Médias seguidas por letras iguais na coluna não diferem entre si pelo Tukey à $5 \%$ de probabilidade.

Para índice de área foliar (IAF), verificou-se diferenças significativas entre os substratos, onde resíduo de soja e palha de arroz foi superior apresentando média 61,67 e 64,09 respectivamente, em relação aos tratamentos mistura e testemunha que apresentaram 32,43 e 32,10, respectivamente. Segundo Dorneles et al. (2019) o IAF pode ser utilizado para representar a eficiência fotossintética, também para analisar o crescimento e como fator condicionante da produtividade, pois através do IAF é definida a capacidade do dossel em interceptar a radiação solar, converter em matéria seca através da fotossíntese e determinar o potencial produtivo da cultura, o que justifica o resultado da produtividade da alface cultivada no substrato resíduo de soja.

Analisando o parâmetro RAF observou-se que o tratamento testemunha a base de areia preta se sobressaiu dos demais, apresentando media $189,52 \mathrm{~cm}^{2} \mathrm{~g}^{-1}$. Henrique et al. (2011), citam que a RAF expressa um componente morfofisiológico que representa uma relação entre a área foliar, esta é responsável pela interceptação da energia luminosa, $\mathrm{CO} 2$ e a massa de matéria seca total que é resultante da fotossíntese.

Com relação a RPF, o tratamento testemunha foi superior em relação aos demais tratamentos com resultado de 231,66. Falqueto et al. (2009) afirmam, os aumentos na razão de massa foliar refletem maior alocação de assimilados para as folhas em desenvolvimento, tidas como drenos metabólicos, e o decréscimo desta razão ao longo do desenvolvimento da planta reflete a mobilização de compostos fotoassimilados para outros órgãos da planta. Segundo Casais et al. (2020) o aumento da RPF indica que a proporção da biomassa que está retida nas folhas e a exportada para outras partes depende da interceptação de radiação solar, bem como o sistema em que a planta está inserida.

Para o índice de área foliar especifica (AFE) constatou-se que o substrato testemunha foi superior aos tratamentos palha de arroz, areia preta e mistura apresentando $190,32 \mathrm{~cm}^{2} \mathrm{~g}^{-1}$. No entanto observando o parâmetro PEF e QAPA verifica-se que o substrato resíduo de soja foi superior aos demais tratamentos com resultados de $0,018 \mathrm{~g} \mathrm{~cm}^{2}$ e 647,70 respectivamente.

A cultivar veneranda apresenta um ciclo de 60 a 70 dias de acordo com informações descritas pelo fornecedor das sementes, nesse experimento entre a semeadura e a colheita o ciclo foi de 51 dias para o 
substrato resíduo de soja, 59 para palha de arroz e 65 para testemunha e mistura. Lima et al. (2018), observou que o ciclo da cultivar Veneranda foi de 67 dias, desta forma observa-se que está a cultivar apresentou um ciclo reduzido com o cultivo em substratos e ambiente protegido demonstrando ser uma alternativa promissora para produção de alface.

\section{CONCLUSÕES}

O melhor substrato para produção de alface em casa de vegetação é o resíduo de soja, pois nesse estudo a cultivar Veneranda apresentou a maior produtividade e os melhores índices morfofisiológicos. Demostrando a possibilidade da utilização desse resíduo industrial, como substrato alternativo.

AGRADECIMENTOS: A UFRA pela concessão da bolsa de PIBIC a primeira autora.

\section{REFERÊNCIAS}

ALENCAR, T. A.; TAVARES, A. T.; CHAVES, P. P. N.; FERREIRA, T. A.; NASCIMENTO, I. R.. Efeito de intervalos de aplicação de urina bovina na produção de alface em cultivo protegido. Revista Verde. Mossoró, v.7, n.3, p.53-67, 2012.

AVIZ, R. O.; BORGES, L. S.; SILVA, M. J. S.; CASAIS, L. K. N.; CARMO, A. S.; SOARES, D. S.; SILVA, F. C. G.; CARVALHO, F. S.. Adaptação de cultivares de alface (Lactuca sativa L.) ás condições climáticas do sudeste paraense. In: Livro Grandes temas em agronomia. 2019. p.141.

AZEVEDO, L. C.; OLIVEIRA, A. C.; MARTINS, I. C. S.; SILVA, V. L.; RIBEIRO, C. S.. Salinidade do solo em ambiente protegido. Rev. Ciências Exatas e da Terra e Ciências Agrárias, Campo Mourão, v.13, n.1, p.52-69, 2018.

BEZERRA, M. D. L.. Cinza vegetal como corretivo e fertilizante no cultivo de capim-marandu em solos do cerrado Mato-grossense. Dissertação (Mestrado em Engenheira Agrícola) - Universidade Federal de Mato Grosso, Rondonópolis, 2013.

BLANCO, F. F.; FOLEGATTI, M. V.; HENRIQUES NETO, D.. Doses de $\mathrm{N}$ e $\mathrm{K}$ no tomateiro sob estresse salino: I. Concentração de nutrientes no solo e na planta. Revista Brasileira de Engenharia Agrícola e Ambiental, v.12, n.1, p.26-33, 2008.

CABRAL, M. B. G.; SANTOS, G. A.; SANCHEZ, S. B.; LIMA, W. L.; RODRIGUES, W. N.. Avaliação de substratos alternativos para produção de mudas de alface utilizados no sul do Estado do Espírito Santo. Revista Verde de Agroecologia e Desenvolvimento Sustentável, Mossoró, v.5, n.1, p.43-48, 2011.

CASAIS, L. K. N.; BORGES, L. S.; AVIZ, R. O.; MATOS JUNIOR, F. T.; LIMA, M. S. S.; SOUSA, V. Q.. Aproveitamento de resíduo de soja e palha de arroz como substrato para produção de mudas de Jambu. Cadernos de Agroecologia. Brasília, v.13, n.1, p.1-6, 2018.

CASAIS, L. K. N.; BORGES, L. S.; MEDEIROS, M. B. C. L.; SOUZA, M. E.; SOARES, D. S.. Índices morfofisiológicos e clorofila de Hortelã-pimenta cultivadas sob diferentes sistemas de cultivos. Revista Ibero-Americana de Ciências Ambientais, Aracaju, v. 1, n.3, 2020.

DORNELES, M. M.; BRITO, G. H. M.. Índice de área foliar e suas aplicações na agricultura. In: SEMANA AGRONÔMICA TECNOLOGIA E SUSTENTABILIDADE NA AGROPECUÁRIA DO CERRADO, 8. Anais. Goianésia: Seagro, 2019. p.1-1.

EMBRAPA. Centro Nacional de Pesquisa de Solos. Sistema Brasileiro de Classificação de Solos. Brasília: Embrapa Solos, 2018.

FALQUETO, A. R.; CASSOL, D.; MAGALHÃES JÚNIOR, A. M.; OLIVEIRA, A. C.; BACARIN, M. A.. Crescimento e partição de assimilados em cultivares de arroz diferindo no potencial de produtividade de grãos. Bragantia, v.68, p.563-571, 2009.

FERREIRA, D. F.. Sisvar: a computer statistical analysis system. Revista Ciência e Agrotecnologia, Lavras, v.35, n.6, p.1039-1042, 2011.

FISCH, G.; MARENGO, J. A.; NOBRE, C. A.. Clima da Amazônia. Climanálise, 1996.

FREITAS, G. A.; SILVA, R. R.; BARROS, H. B.; VAZ-DE-MELO, A.; ABRAHÃ, W. A. P.. Produção de mudas de alface em função de diferentes combinações de Substratos. Revista Ciência Agronômica, v.44, n.1, p.159-166, 2013.

HENRIQUE, P. C.; ALVES, J. D.; DEUNER, S.; GOULART, P. F. P.; LIVRAMENTO, D. E.. Aspectos fisiológicos do desenvolvimento de mudas de café cultivadas sob telas de diferentes colorações. Pesq. Agropec. Bras, Brasília, v.46, n.5, p.458-465, 2011.

IBARRA, L.; FLORES, J.; PÉREZ, J. C. D.. Growth and yield of muskmelon in response to plastic mulch and row covers. Scientia Horticulturae, Coah, v.87, n.1-2, p.139-145, 2001.

LIMA, M. S. S.; BORGES, L. S.; SANTOS, N. F. A.; MELO, M. R. S.; SOUSA, V. Q.; BIRANI, S. M.; PEDROSO, A. J. S.; GOMES, R. F.. Qualidade e produtividade econômica de cultivares alface conduzidas nas condições edafoclimáticas do Sudeste Paraense. Agroecossistemas, v.10, n.1, p.227-240, 2018. 
LODOLINI, E. M.; PICA, F.; MASSETANI, F.; NERI, D.. Physical, Chemical and Biological Properties of some Alternative Growing Substrates. International Journal of Soil Science, 12: 32-38. 2017.

MAGGI, M. F.; KLAR, A. E.; JADOSKI JUNIOR, C.; ANDRADE, A. R. S.. Produção de variedades de alface sob diferentes potenciais de água no solo em ambiente protegido. Irriga, v.11, p.415-427, 2006

PEREIRA JUNIOR, A. S.; SOARES, D. S.; ALVES, G. A. R.; BORGES, L. S.; FREITAS, L. S.; SOUZA, M. E.. Produtividade e análise sensorial de cultivares de alface (Lactuca sativa L.) hidropônica em período chuvoso e seco de Paragominas Pa. In: SANTOS, C. A.. Pesquisa e inovação em horticultura. Maringá: Uniedusul, 2020. p.5-16.

PURQUERIO, L. F. V.; DEMANT, L. A. R.; GOTO, R.; BOAS, R. L.
V.. Efeito da adubação nitrogenada de cobertura e do espaçamento sobre a produção de rúcula. Horticultura Brasileira, v.25, n.3, p.464-470, 2007.

RODRIGUES, T. E.; VALENTE, M. A.; GAMA, J. R. N. F.; OLIVEIRA JÚNIOR, R. C.; SANTOS, P. L.; SILVA, J. L.. Zoneamento Agroecológico do município de Paragominas, Estado do Pará. Belém: Embrapa Amazônia Oriental, 2002.

SOUZA, A. L.; SEABRA JÚNIOR, S.; DIAMANTE, M. S.; SOUZA, L. H. C.; NUNES, M. C. M.. Comportamento de cultivares de alface americana sob clima tropical. Revista Caatinga, v.26, n.4, p.123-129, 2013.

YURI, J. E.; MOTA, J. H.; DE RESENDE, G. M.; SOUZA, R. J. Nutrição e adubação da cultura da alface. IN: PRADO, R. M; CECÍLIO FILHO, A. B.. Nutrição e adubação de hortaliças. Jaboticabal: FCAV, 2016. p.559-577. 